Original Article

\title{
EFFECT OF TOCOTRIENOL PRETREATMENT ON EX VIVO SUPEROXIDE AND PEROXIDE HANDLING CAPACITIES (SPHC) OF RAT SERUM AND BRAIN
}

\author{
PITCHAIAH DASARI, ANANDAMURALI R., PRASUNPRIYA NAYAK
}

Department of Physiology, NRI Medical College and General Hospital, Chinakakani, Guntur District, Andhra Pradesh 522503

Email: nayak@nrias.ac.in

Received: 25 Oct 2016 Revised and Accepted: 17 Jan 2017

\section{ABSTRACT}

Objective: Tocotrienol (TT), a constituent of vitamin E, present only in selected seed oil. Because of the isoprenoid side chain, antioxidant property of tocotrienol is recently highlighted. Application of tocotrienol is also proven to be neuroprotective. The current study was aimed to evaluate the effect of tocotrienol pretreatment on the serum and brain oxidative stress parameters and oxidant handling capacities.

Methods: Male albino Wistar rats were treated with tocotrienol (10 mg/day) for two weeks and maintained for the next four weeks. Levels of reduced glutathione and lipid peroxidation and activities of superoxide dismutase, catalase, glutathione peroxidase and glutathione reductase were estimated fortnightly in serum. After sacrifice, oxidative stress parameters were measured in the frontal cortex, temporal cortex, thalamic area, hippocampus and cerebellum. Glutathione-dependent and glutathione-independent superoxide and peroxide handling capacities (SPHC) were calculated for serum and brain regions. Data collected from both the groups are statistically processed with Kruskal-Wallis test and Mann-Whitney pairwise comparisons.

Results: Significant impacts of TT treatment have been observed in terms of growth and water intake. Serum SPHC (Glutathione-independent) has been found to be reduced significantly immediately after the TT treatment. Region-specific alterations in oxidative stress parameters have also been observed after $4 \mathrm{w}$ of supplementation. Global reductions in reduced glutathione and lipid peroxidation have been observed in the brain without any alteration in the SPHC.

Conclusion: From the results, it can be suggested that the tocotrienol pretreatment possibly be used as neuroprotective measure particularly against oxidative stress. In addition, the antioxidant impacts of TT were found to be maintained for a longer period in brain regions, even though it was not so in the case of serum.

Keywords: Tocotrienol, Reduced glutathione, Lipid peroxidation, Superoxide dismutase, Catalase, Glutathione peroxidase, Glutathione reductase, Superoxide and peroxide handling capacity (SPHC), Frontal cortex, Temporal cortex, Thalamic area, Hippocampus, Cerebellum

(C) 2016 The Authors. Published by Innovare Academic Sciences Pvt Ltd. This is an open access article under the CC BY license (http://creativecommons.org/licenses/by/4. 0/) DOI: http://dx.doi.org/10.22159/ijpps.2017v9i3.15866

\section{INTRODUCTION}

Tocotrienols (TT) are the tocopherol homologues having isoprenoid side chain. Like the tocopherols, TTs are noted antioxidants; however, the antioxidant potential of TTs is higher than that of tocopherols while availability in natural seed oil products is quite lesser than the tocopherols. Apart from this, the TTs are reported to oppose inflammation, angiogenesis, cancer, hypercholesterolemia, etc. and interestingly, these effects are not reliant on the antioxidant activity of TT [1]

It has been suggested that the antioxidative effects of TT are due to its capacity to induce specific antioxidant enzymes like superoxide dismutase (SOD), NADPH: Quinone oxidoreductase (NQO) and glutathione peroxidase (GPx) and thereby mediate the quenching of free radicals [2] and removal of lipo peroxyl radicals [3]. Supporting the notion of TTs being better antioxidants compared to tocopherols, Ahsan et al. [3] also indicated the importance of the inferiority of TTs in terms of bioavailability after oral intake. Nevertheless, there are reports documenting antioxidant activities of TT after oral supplementation. Azlina et al. [4] demonstrated that oral supplementation of TT reduced the levels of lipid peroxidation in the stomach, while, Reznick et al. [5] noted suppression of protein oxidation in skeletal muscle and provided evidence for systemic antioxidant effects of TT.

Neurons are particularly sensitive to oxidative stress and their functional terms depend on the oxidant status of the local microenvironment $[6,7]$. On the other hand, because of their unsaturated side chain, penetration into brain tissues is easier for TT [8]. Hence, oral supplementation of TT could be effective in protection of the brain against oxidative stress. At the same time, the quick turnover of plasma TT and lower availability of dietary supplemented TT to brain raised the question of its effectiveness as an antioxidant in the brain.

Accordingly, the present work was aimed to evaluate the antioxidant activity of orally supplemented TT on the serum and brain oxidative stress parameters. Repeated evaluations of serum oxidative stress parameters and one-time evaluation of regional oxidative stress parameters in the brain were carried out in rats supplemented orally with TT. In addition, superoxide and peroxide handling capacities of serum and brain were also calculated to understand the impact of TT supplementation on systemic and neuronal reserve power to withstand oxidative stress particularly when there was no greater than usually encountered oxidant threat.

\section{MATERIALS AND METHODS}

\section{Materials}

Oryza tocotrienol@- -90 was kindly donated by the Oryza Oil and Fat Chemical Co. Ltd, Japan. All other reagents were of analytical grade and procured from Sigma, SRL, SDS, Merck, HiMedia.

\section{Animal maintenance and treatment}

The experimental protocol was approved by the Institutional Animal Ethics Committee (Lt. No. 43/Chairman-IAEC, NRI Medical College and GH, Chinakakani; Dated 16-06-2014). Male albino Wistar rats weighing 120-140 g were obtained, maintained and treated in the Central Animal House of NRI Medical College and General Hospital, and the procedures were performed according to the guidelines of the Committee for the Purpose of Control and Supervision of Experiments on Animals (CPCSEA, India). After one week of 
acclimatization, rats were randomly divided (with the help of Random Allocation Software Version 1.0, May 2004) into two groups-TT+[exposed to Oryza-tocotrienol(C-90 (10 mg/day) orally for two weeks] and $\mathrm{TT}_{0}$ [exposed to sham treatment with distilled water for two weeks].

\section{Serum collection and Biochemical assays}

Nearly $1 \mathrm{ml}$ of blood was collected through retro-orbital puncture in four occasions: (i) prior to the initiation of exposures [Week (-2)], (ii) at the end of two weeks of exposures [Week 0], (iii) at the end of $2 \mathrm{w}$ of post-exposure [Week 2] and (iv) at the end of $4 \mathrm{w}$ of postexposure [Week 4/sacrifice]. Overnight fasted rats were sacrificed by cervical dislocation. The whole brain was removed, washed with ice-cold saline. Under the dissection microscope, frontal cortex (FC), temporal cortex (TC), thalamic area (TH), hippocampus (HC) and cerebellum (CL) were immediately separated following Chiu et al. (2007) [leftover parts of the brain were collected together as rest of the brain (RB)] and preserved in the deep fridge for biochemical estimations.

Brain tissues were homogenized in the required buffer and centrifuged at $1000 \mathrm{rpm}$ in cold $\left(4^{\circ} \mathrm{C}\right)$ for $5 \mathrm{~min}$. Protein levels of the supernatants are measured by modified Lowry method [9]. The regional content of reduced glutathione (GSH) was measured following the method of Griffith [10]. The weighed tissue pieces were homogenized in $1.5 \mathrm{ml}$ of ice-cold $0.1 \mathrm{M}$ phosphate buffer $(\mathrm{pH}$ 7.4). Then, $200 \mu \mathrm{L}$ homogenate were immediately mixed with $200 \mu \mathrm{L}$ $4 \%(\mathrm{w} / \mathrm{v})$ sulfosalicylic acid. After shaking well, the mixtures were centrifuged at $3000 \mathrm{rpm}$ for $10 \mathrm{~min}$. The $100 \mu \mathrm{L}$ aliquot of the supernatant was mixed with $3.0 \mathrm{ml}$ of 5,5'-dithiobis (2-nitrobenzoic acid) (DTNB; $0.1 \mathrm{mmol}$, in 0.01M phosphate buffer, pH 8.0) and after 2 min, absorbances were recorded at $412 \mathrm{~nm}$. Using gradually varied concentrations of glutathione which were treated with DTNB in the same manner as described above, a standard curve of GSH was prepared and used for calibration of the calculation of regional GSH content.

Level of lipid peroxidation was estimated by measuring the level of malonaldehyde or malondialdehyde (MDA) in samples with the help of thiobarbituric acid (TBA). Brain tissue homogenates (in $0.1 \mathrm{M}$ phosphate buffer containing 1 mmol EDTA, pH 7.4) were used to determine the level of MDA production following the method described by Buege and Aust [11]. To $500 \mu \mathrm{L}$ of homogenate sample, $1 \mathrm{ml}$ of $10 \%$ TCA was added followed by $2 \mathrm{ml}$ of $0.67 \%$ thiobarbituric acid (TBA). The mixtures were heated in a water bath at $80^{\circ} \mathrm{C}$ (instead of $100^{\circ} \mathrm{C}$ to minimize the interference of some carbohydrate) for $15 \mathrm{~min}$. After cooling and centrifugation at 3000 rpm for $10 \mathrm{~min}$, the absorbance of the supernatants were read at $535 \mathrm{~nm}$. A reagent blank was prepared following the same procedure using water instead of a sample. The extent of lipid peroxidation was expressed as nmol MDA formed in the processed sample using the molar extinction coefficient for MDA of $1.56 \times 10^{5}$ $\mathrm{M}^{-1} \mathrm{~cm}^{-1}[12]$.

The SOD activity was measured by the kinetic study measuring inhibition of auto-oxidation of pyrogallol. Initially, auto-oxidation of pyrogallol was measured by using the solution of $0.2 \mathrm{mmol}$ pyrogallol in air equilibrated $50 \mathrm{mmol}$ Tris-HCl buffer $(\mathrm{pH} 8.2)$ containing $1 \mathrm{mmol}$ diethylene-triamine-pentaacetic acid. The rate of auto-oxidation was taken from the increase in absorbance at 420 $\mathrm{nm}$. The percentage inhibition of the rate of auto-oxidation of pyrogallol was measured by addition of the sample. Enzyme activity which produced an inhibition of pyrogallol auto-oxidation by $50 \%$, considered as one unit and accordingly the SOD activities of brain regions are expressed in Units [13].

The activity of catalase was assayed by tracking decomposition of hydrogen peroxide in a system contained $1.90 \mathrm{ml}$ of $0.05 \mathrm{M}$ sodium phosphate buffer $\mathrm{pH} 7.0,0.10 \mathrm{ml}$ enzyme source (containing 20-40 $\mu \mathrm{g}$ protein) and $1.0 \mathrm{ml}$ of $0.05 \mathrm{M}$ Hydrogen peroxide in phosphate buffer. The change in absorbance was read at $240 \mathrm{~nm}$ at regular intervals. The specific activity of catalase enzyme was calculated using molar extinction coefficient of $\mathrm{H}_{2} \mathrm{O}_{2}$ as $43.6 \mathrm{M}^{-1} \mathrm{~cm}^{-1}$ [14].

The activity of GPx for each sample was assayed as per the following protocol. Brain tissue homogenate $(0.1 \mathrm{ml})$ was added to $2.58 \mathrm{ml}$ of $0.05 \mathrm{M}$ sodium phosphate buffer ( $\mathrm{pH} 7.0$ ) containing $0.005 \mathrm{M}$ EDTA. Following solutions were added in turn: $0.1 \mathrm{ml}$ of $0.0084 \mathrm{mmol}$ $\mathrm{NADPH}, 0.01 \mathrm{ml}$ of Yeast glutathione reductase (Sigma chemicals; contains 200 units $/ \mathrm{ml}$ ), $0.01 \mathrm{ml}$ of $1.125 \mathrm{M}$ sodium azide and $0.1 \mathrm{ml}$ of $0.15 \mathrm{M}$ reduced glutathione. The reaction mixture was allowed to equilibrate. The enzymatic reaction was initiated by addition of 0.1 $\mathrm{ml}$ of $0.0022 \mathrm{M}$ hydrogen peroxide (prepared by $50 \mathrm{mmol}$ sodium phosphate buffer, $\mathrm{pH}$ 7.0). The conversion of NADPH to NADP+tracked by continuous (every 10 seconds) recording of the change in absorbance of the system at $340 \mathrm{~nm}$ between 2-4 min after initiation of the reaction. A reagent blank was prepared using buffer instead of tissue homogenate. The specific activity of GPx was calculated using the extinction coefficient of $6.22 \mathrm{~cm}^{-1} / \mu \mathrm{mol}$ for NADPH [15].

Activities of GR in test samples were assayed by monitoring the use of NADPH during conversion of GSSG to GSH. The assay system contained $0.25 \mathrm{ml}$ of $0.084 \mathrm{M}$ Tris- $\mathrm{HCl}$ buffer $\mathrm{pH} 7.4,0.05 \mathrm{ml}$ of 150 mmol EDTA, $0.05 \mathrm{ml}$ of $9.0 \mathrm{mmol} \mathrm{NADPH}, 0.48 \mathrm{ml}$ distilled water and $0.05 \mathrm{ml}$ of enzyme source containing $50-80 \mu \mathrm{g}$ protein. The reaction was initiated by addition of $0.1 \mathrm{ml}$ of $50 \mathrm{mmol}$ oxidized glutathione (GSSG). The change in absorbance was recorded at 1 min interval at $340 \mathrm{~nm}$. The specific activity of the enzyme was expressed as nmol of NADPH oxidized/unit time using the extinction coefficient for NADPH of $6.22 \mathrm{~cm}^{2} / \mu$ mole [16].

Superoxide dismutase is the enzyme responsible for the conversion of superoxide into hydrogen peroxide. The hydrogen peroxide thus produced, is still remaining as a threat for the cytosolic environment (fig. 1). Therefore, individual study of SOD activity provides an only partial clue for the cellular mechanisms against pro-oxidant condition. On the other hand, conversion of hydrogen peroxide into water is carried out by two major systems, (a) glutathione peroxidase system, which uses glutathione as a donor of reducing equivalent, and (b) catalase system, which does not need glutathione as a donor of reducing equivalent. These two steps are complementary to each other in regard to handling the oxidative power superoxide. Therefore, the regional capacity of handling superoxide and peroxide can be estimated by the ratio of performance of these two steps of oxidant reduction or superoxide neutralisation [17].

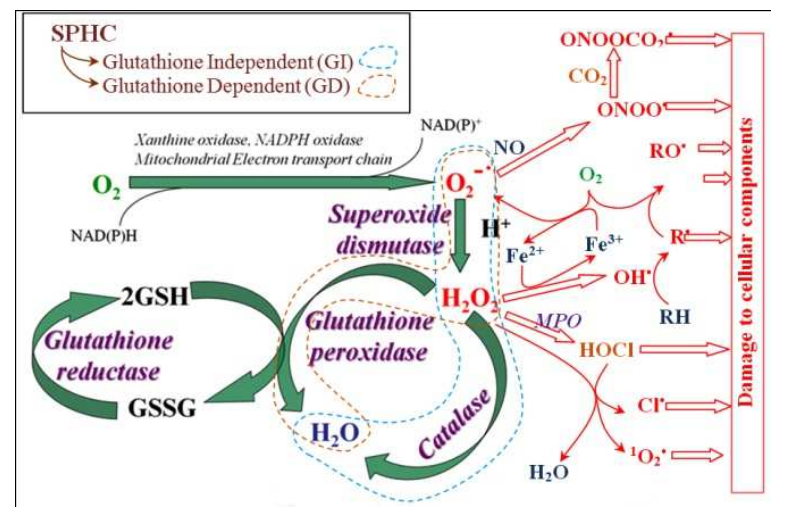

Fig. 1: Pathways of oxidative stress with enzymes involved in superoxide peroxide handling capacities (SPHC) [17] 
To evaluate the capacity of serum and brain samples in regard to withstanding oxidative stress and minimise the impacts of the threat of oxidant imbalance, superoxide and peroxide handling capacity of each brain regions were calculated (fig. 1). The ratio of activities of catalase and SOD, expressed in terms of the equal amount of brain tissue or tissue protein, were calculated and represented as glutathione-independent superoxide and peroxide handling capacity (SPHC-GI). Similarly, the ratios of GPx and SOD activities were expressed as glutathione-dependent superoxide and peroxide handling capacity (SPHC-GD).

\section{Statistical analyses}

Variance and difference between the groups are analyzed by Kruskal-Wallis (KW) test and Mann-Whitney pairwise comparisons (MW), respectively, accepting the probability of $5 \%$ or less as significant using PAST statistical software (ver. 3.12; Copyright: $\varnothing$. Hammer 1999-2016) [18].

\section{RESULTS}

The body weights of rats were regularly monitored and the changes in growth rate for both groups of rats are presented in fig. 2. Significant Tie corrected $\chi^{2}$ value indicates that substantial influence of TT supplementation has been noted in terms of growth rate. However, the growth rate of TT+group is significantly differing from that of $\mathrm{TT}_{0}$ group only in week 2 . By the fourth week after the supplementation, both groups demonstrated a sharp decline in their growth rate, which could be due to the age effect. Though average feed taken by both groups were not differing throughout the study, a trend of higher water intake in $\mathrm{TT}_{+}$group has been noted (fig. 3).
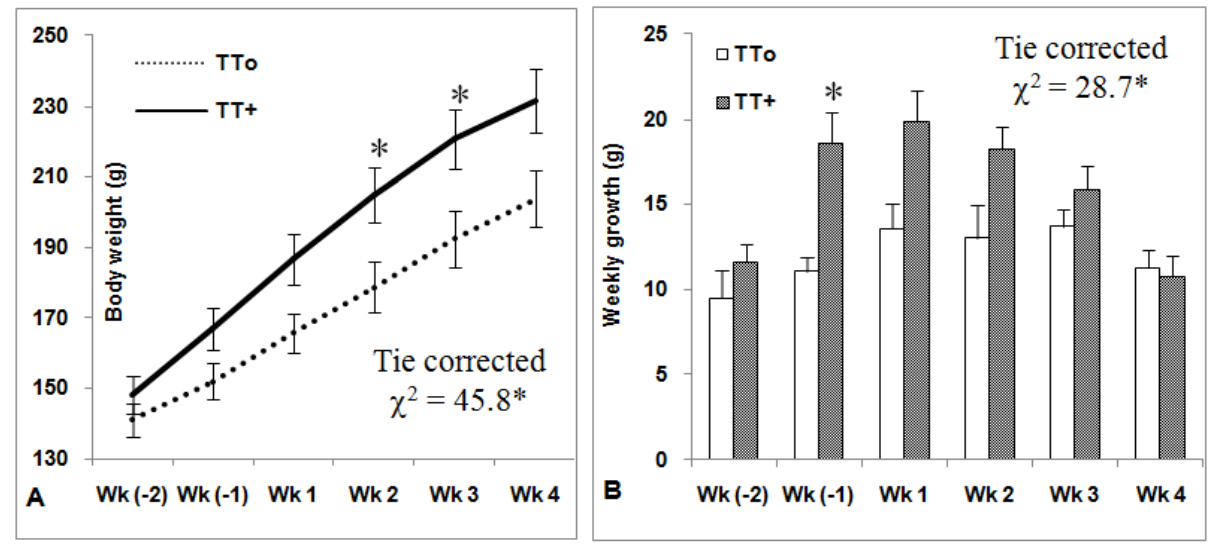

Fig. 2: Changes in body weight (A) and weekly growth (B) of $\mathrm{TT}_{0}$ and $\mathrm{TT}_{+}$rats during the study period. Each column/data point represents mean of five observations \pm Standard Error of Mean * indicates $p<0.05$ (Difference between $\mathrm{TT}_{+}$and $\mathrm{TT}_{0}$ in case of weekly values)

None of the tested biochemical parameters for evaluation of oxidative stress-GSH, TBARS, Catalase, SOD, has demonstrated a significant difference between the $\mathrm{TT}_{+}$and $\mathrm{TT}_{0}$ groups in all the four occasions of serum collections (table 1).

Table 1: Ex vivo values of some oxidative stress parameters of serum collected fortnightly during the study period

\begin{tabular}{|c|c|c|c|c|c|}
\hline & Animal groups & Reduced glutathione $^{a}$ & Lipid peroxidation $^{b}$ & Catalase $^{c}$ & Superoxide dismutase $^{d}$ \\
\hline \multirow[t]{2}{*}{ Week $(-2)$} & $\mathrm{TT}_{0}$ & $64.80 \pm 17.41$ & $52.84 \pm 6.69$ & $14.91 \pm 1.29$ & $32.38 \pm 2.97$ \\
\hline & $\mathrm{TT}_{+}$ & $63.60 \pm 6.13$ & $60.97 \pm 1.66$ & $14.53 \pm 5.05$ & $37.26 \pm 1.85$ \\
\hline \multirow[t]{2}{*}{ Week 0} & $\mathrm{TT}_{0}$ & $56.19 \pm 16.60$ & $68.48 \pm 16.14$ & $11.93 \pm 1.17$ & $30.76 \pm 3.21$ \\
\hline & $\mathrm{TT}_{+}$ & $54.61 \pm 9.81$ & $49.93 \pm 7.02$ & $5.99 \pm 1.75$ & $36.54 \pm 2.27$ \\
\hline \multirow[t]{2}{*}{ Week 2} & $\mathrm{TT}_{0}$ & $52.67 \pm 8.77$ & $65.02 \pm 9.55$ & $11.97 \pm 1.17$ & $33.60 \pm 2.88$ \\
\hline & $\mathrm{TT}_{+}$ & $53.13 \pm 10.11$ & $48.42 \pm 2.61$ & $13.06 \pm 5.60$ & $33.47 \pm 5.38$ \\
\hline \multirow[t]{2}{*}{ Week 4} & $\mathrm{TT}_{0}$ & $49.09 \pm 3.46$ & $59.50 \pm 9.57$ & $11.75 \pm 1.12$ & $30.71 \pm 0.92$ \\
\hline & $\mathrm{TT}_{+}$ & $47.62 \pm 5.84$ & $56.80 \pm 9.06$ & $9.92 \pm 2.00$ & $33.38 \pm 2.44$ \\
\hline \multicolumn{2}{|c|}{ Tie-corrected $\chi^{2}$ value } & 3.214 & 7.127 & 10.59 & 7.361 \\
\hline
\end{tabular}

Data are mean \pm Standard Error of Mean of 5 observations (n). ${ }^{a}=\mu \mathrm{g} / 100$ mg protein, ${ }^{b}=\mu$ mol $/ 100$ mg protein, ${ }^{c}=\mu m_{0}$ le $\mathrm{H}_{2} \mathrm{O}_{2} / \mathrm{hr} / 100 \mathrm{mg}$ protein, ${ }^{\mathrm{d}}$ = Units $/ 100 \mathrm{mg}$ protein.

Similarly, activities of serum GPx and GR also remain unchanged throughout the study period (table 2). Only the glutathioneindependent SPHC of serum is found to be significantly lower in the $\mathrm{TT}_{+}$group during week 0 evaluation; however, without any change in glutathione-dependent SPHC of serum (table 2).

Though all the brain regions have demonstrated a significant reduction in GSH contents, only the decrements in TH and RB are found to be significant as per KW test (fig. 4). Similarly, reductions in TBARS level of FC, TH and $\mathrm{HC}$ are proved to be significant by tie corrected $\chi^{2}$ value of KW test, while all other tested regions demonstrated insignificant decreases in the TBARS values (fig. 5). Fig. 6 depicts unaltered catalase activities in all the tested brain regions. Except in TH, SOD activities of all the brain regions indicates a rise in the $\mathrm{TT}_{+}$group; however, the difference with the $\mathrm{TT}_{0}$ group is found to be statistically significant only in RB (fig. 7). Similarly, trends of enhancement of GPx activities are exhibited by all the brain regions without statistical significance (KW test) with an exception in CL where the GPx activity in the TT+group is significantly higher than that of the $\mathrm{TT}_{0}$ group (fig. 8).

Likewise, HC has demonstrated significant increment in GR activity in the $\mathrm{TT}_{+}$group while all other brain regions have recorded only insignificant increment in GR activities (fig. 9). Figs. 10 and 11 have depicted the unaltered status of glutathione-independent and glutathione-dependent SPHC in all the tested brain regions. 
Table 2: Ex vivo values of glutathione turnover and superoxide and peroxide handling capacity (SPHC) of serum collected fortnightly during the study period

\begin{tabular}{|c|c|c|c|c|c|}
\hline & Animal groups & Glutathione peroxidase ${ }^{a}$ & Glutathione reductase $^{a}$ & GI-SPHC & GD-SPHC \\
\hline \multirow[t]{2}{*}{ Week $(-2)$} & $\mathrm{TT}_{0}$ & $9.14 \pm 1.13$ & $12.84 \pm 0.61$ & $0.48 \pm 0.05$ & $0.30 \pm 0.06$ \\
\hline & $\mathrm{TT}_{+}$ & $10.25 \pm 1.46$ & $16.07 \pm 2.98$ & $0.39 \pm 0.14$ & $0.27 \pm 0.03$ \\
\hline \multirow[t]{2}{*}{ Week 0} & $\mathrm{TT}_{0}$ & $10.47 \pm 1.05$ & $12.07 \pm 1.26$ & $0.40 \pm 0.05$ & $0.35 \pm 0.04$ \\
\hline & $\mathrm{TT}_{+}$ & $10.31 \pm 2.49$ & $15.30 \pm 2.66$ & $0.17 \pm 0.05^{*}$ & $0.29 \pm 0.08$ \\
\hline \multirow{2}{*}{ Week 2} & $\mathrm{TT}_{0}$ & $9.83 \pm 0.49$ & $13.03 \pm 0.61$ & $0.37 \pm 0.05$ & $0.30 \pm 0.02$ \\
\hline & $\mathrm{TT}_{+}$ & $8.40 \pm 1.57$ & $13.27 \pm 1.53$ & $0.34 \pm 0.09$ & $0.27 \pm 0.06$ \\
\hline \multirow[t]{2}{*}{ Week 4} & $\mathrm{TT}_{0}$ & $10.99 \pm 0.78$ & $12.13 \pm 0.76$ & $0.38 \pm 0.04$ & $0.36 \pm 0.03$ \\
\hline & $\mathrm{TT}_{+}$ & $11.11 \pm 2.12$ & $16.82 \pm 5.80$ & $0.31 \pm 0.08$ & $0.34 \pm 0.06$ \\
\hline \multicolumn{2}{|c|}{ Tie-corrected $\chi^{2}$ value } & 3.817 & 2.690 & 10.04 & 5.295 \\
\hline
\end{tabular}

Data are mean \pm Standard Error of Mean of 5 observations (n). ${ }^{*}$ indicates significant difference in comparison to TT ${ }_{0}$ group. ${ }^{a}=\mu$ mole NADPH oxidized $/ \mathrm{h} / 100$ mg protein, $\mathrm{GI}=$ Glutathione-independent, $\mathrm{GD}=$ Glutathione-dependent, ${ }^{\mathrm{c}}=\mu$ mole $\mathrm{H}_{2} \mathrm{O}_{2} / \mathrm{h} / 100$ mg protein, ${ }^{\mathrm{d}}=\mathrm{Units} / 100 \mathrm{mg}$ protein .

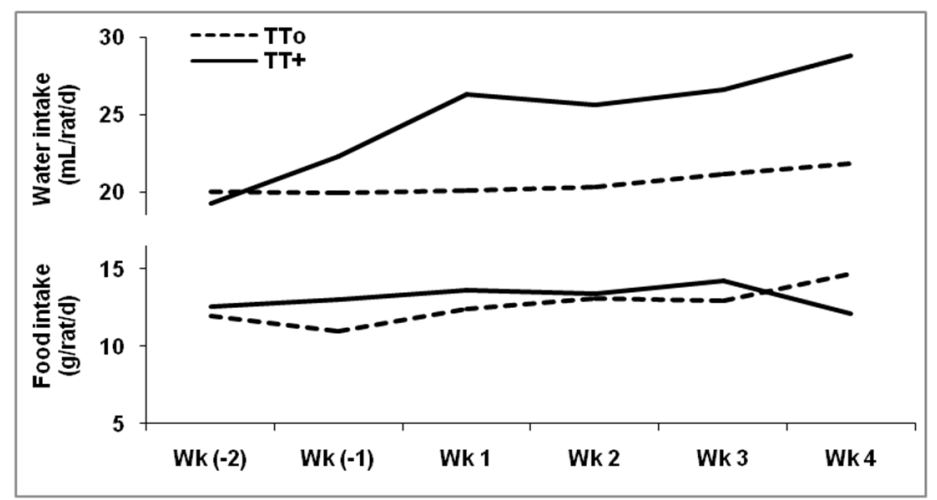

Fig. 3: Changes in weekly average of food and water intakes in $\mathrm{TT}_{0}$ and TT+rats during the study period

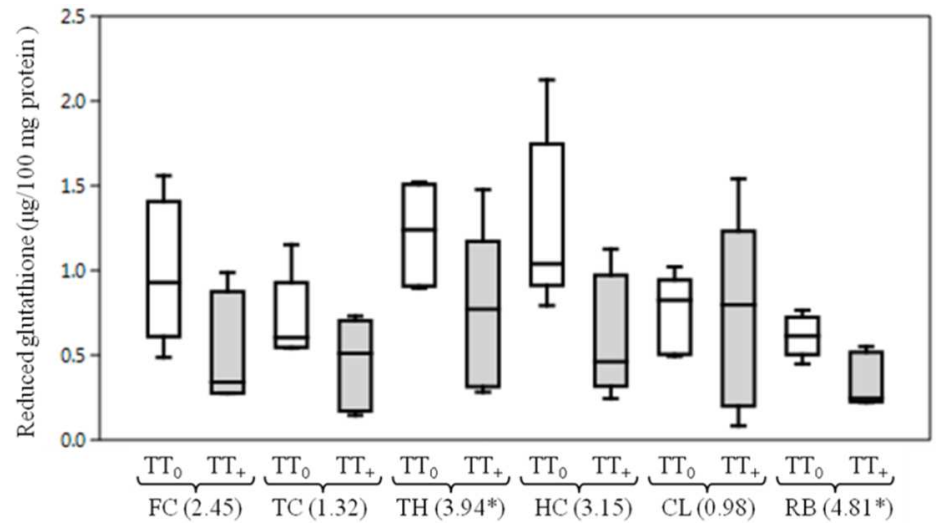

Fig. 4: Box and whisker plots for the levels of reduced glutathione in different brain regions of animal groups. $\mathrm{FC}=\mathrm{Frontal}$ cortex, $\mathrm{TC}=$ Temporal cortex, $\mathrm{TH}=$ Thalamic area, $\mathrm{HC}=$ Hippocampus, $\mathrm{CL}=$ Cerebellum, $\mathrm{RB}=$ Rest of brain. Fig. 4 in parentheses are tie corrected $\chi^{2}$ values obtained by Kruskal-Wallis test $(*=p<0.05)$

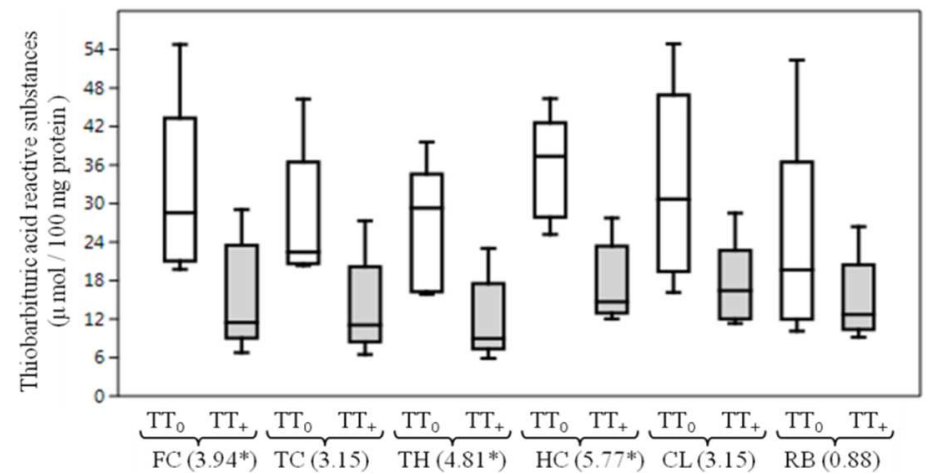

Fig. 5: Box and whisker plots for the levels of lipid peroxidation in different brain regions of animal groups. FC = Frontal cortex, $\mathrm{TC}=$ Temporal cortex, $\mathrm{TH}=$ Thalamic area, $\mathrm{HC}=$ Hippocampus, $\mathrm{CL}=$ Cerebellum, $\mathrm{RB}=$ Rest of brain. Fig. 5 in parentheses are tie corrected $\chi^{2}$ values obtained by Kruskal-Wallis $(*=p<0.05)$ 


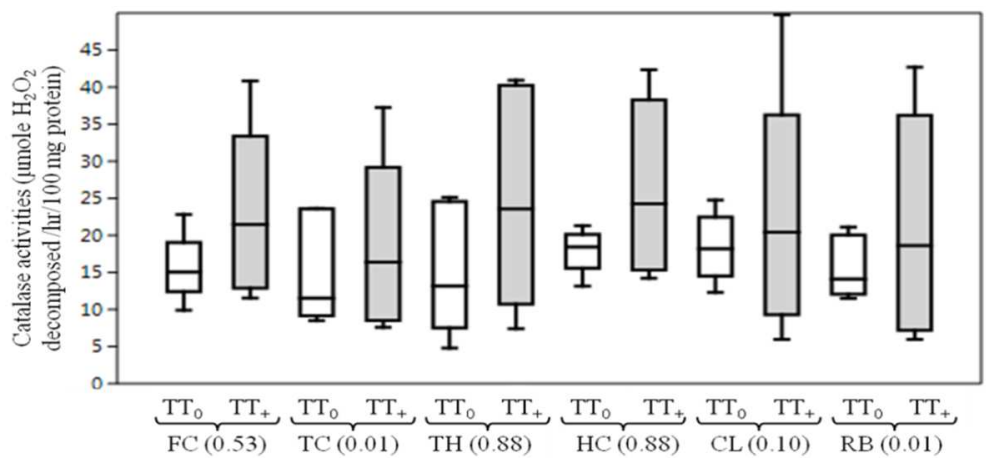

Fig. 6: Box and whisker plots for the catalase activities in different brain regions of animal groups. $\mathrm{FC}=$ Frontal cortex, $\mathrm{TC}=\mathrm{Temporal}$ cortex, $\mathrm{TH}=$ Thalamic area, $\mathrm{HC}=$ Hippocampus, $\mathrm{CL}=$ Cerebellum, $\mathrm{RB}=$ Rest of brain. Fig. 6 in parentheses are tie corrected $\chi^{2}$ values obtained by Kruskal-Wallis test

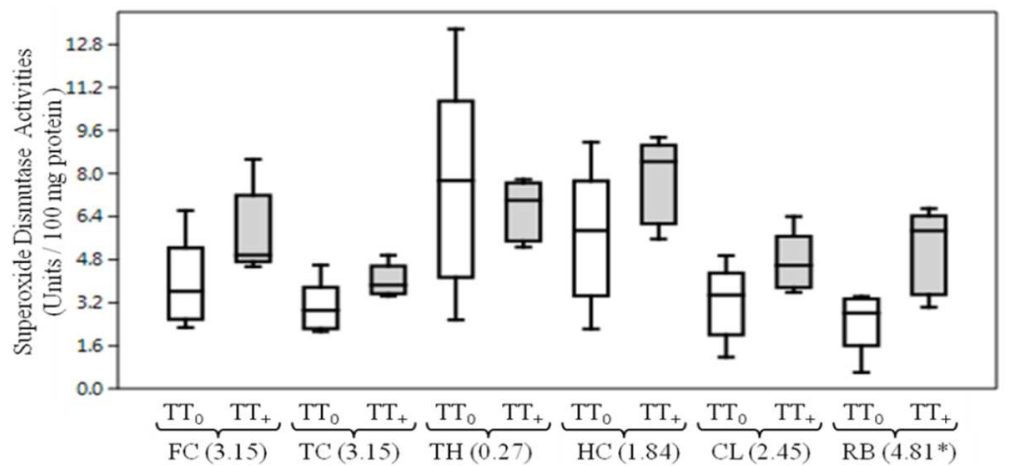

Fig. 7: Box and whisker plots for the superoxide dismutase activities in different brain regions of animal groups. $\mathrm{FC}=\mathrm{Frontal}$ cortex, $\mathrm{TC}=$ Temporal cortex, $\mathrm{TH}=$ Thalamic area, $\mathrm{HC}=$ Hippocampus, $\mathrm{CL}=$ Cerebellum, $\mathrm{RB}=$ Rest of brain. Fig. 7 in parentheses are tie corrected $\chi^{2}$ values obtained by Kruskal-Wallis $(*=p<0.05)$

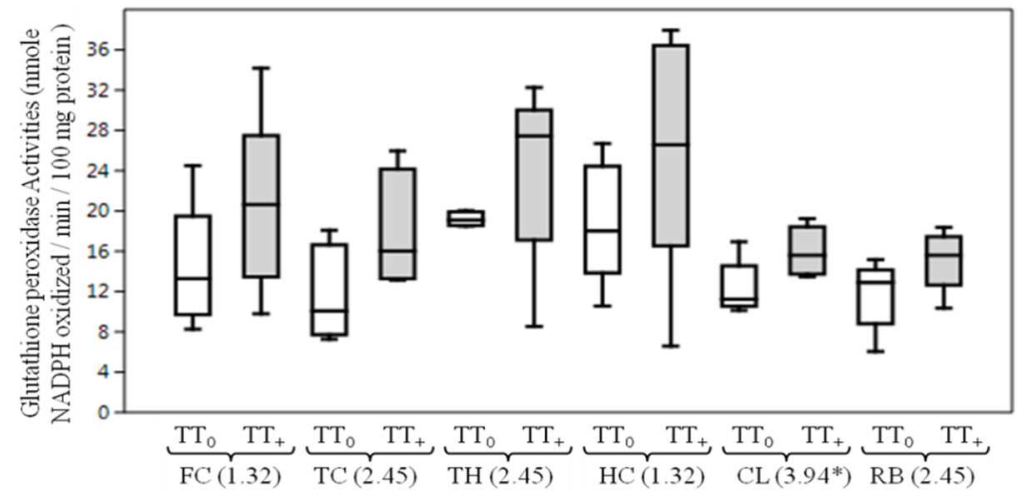

Fig. 8: Box and whisker plots for the glutathione peroxidase activities in different brain regions of animal groups. $\mathrm{FC}=\mathrm{Frontal}$ cortex, $\mathrm{TC}=$ Temporal cortex, $\mathrm{TH}=$ Thalamic area, $\mathrm{HC}=$ Hippocampus, $\mathrm{CL}=$ Cerebellum, $\mathrm{RB}=$ Rest of brain. Fig. 8 in parentheses are tie corrected $\chi^{2}$ values obtained by Kruskal-Wallis $(*=p<0.05)$

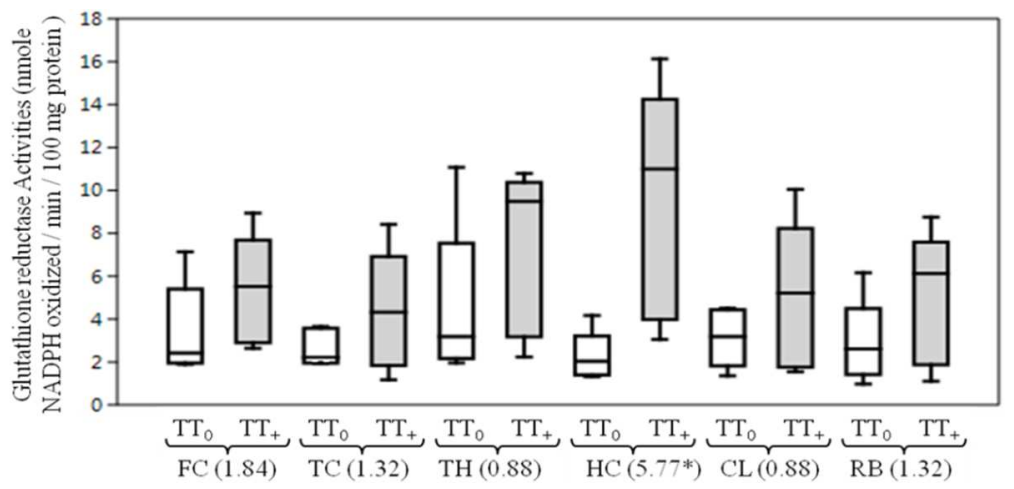

Fig. 9: Box and whisker plots for the glutathione reductase activities in different brain regions of animal groups. FC $=$ Frontal cortex, $\mathrm{TC}=$ Temporal cortex, $\mathrm{TH}=$ Thalamic area, $\mathrm{HC}=$ Hippocampus, $\mathrm{CL}=$ Cerebellum, $\mathrm{RB}=$ Rest of brain. Fig. 9 in parentheses are tie corrected $\chi^{2}$ values obtained by Kruskal-Wallis $(*=p<0.05)$ 


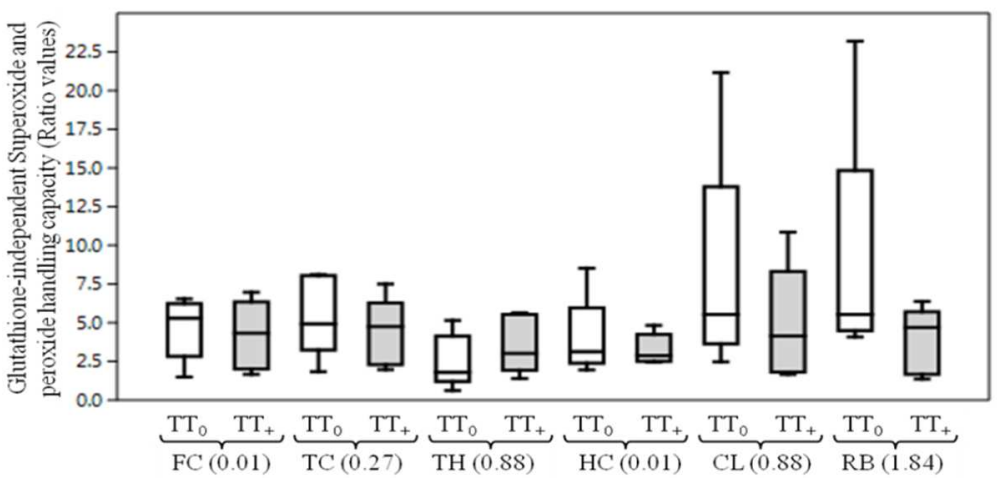

Fig. 10: Box and whisker plots for the GI-SPHC in different brain regions of animal groups. $\mathrm{FC}=$ Frontal cortex, $\mathrm{TC}=\mathrm{Temporal}$ cortex, $\mathrm{TH}=$ Thalamic area, $\mathrm{HC}=$ Hippocampus, $\mathrm{CL}=$ Cerebellum, $\mathrm{RB}=$ Rest of brain. Fig. 10 in parentheses are tie corrected $\chi^{2}$ values obtained by Kruskal-Wallis

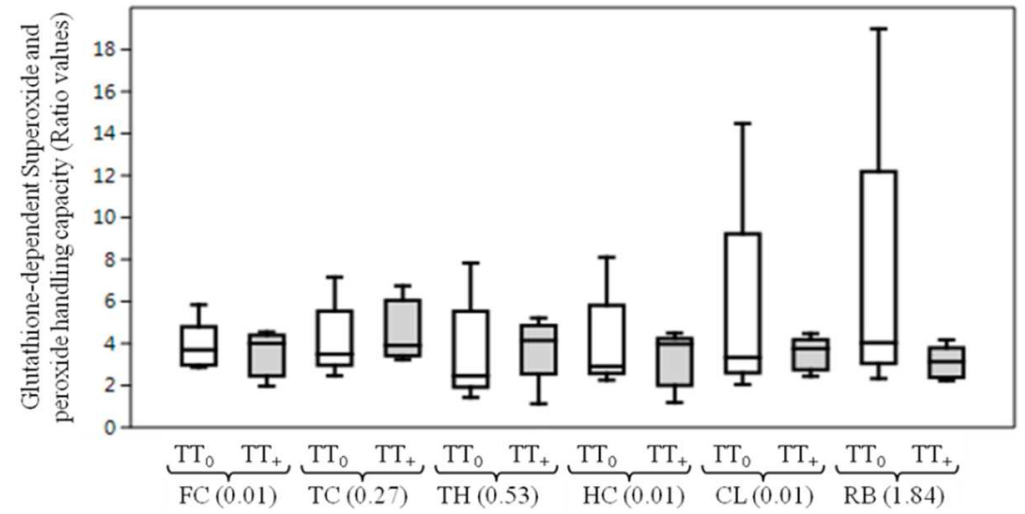

Fig. 11: Box and whisker plots for the GD-SPHC in different brain regions of animal groups. FC = Frontal cortex, $\mathrm{TC}=\mathrm{Temporal}$ cortex, $\mathrm{TH}$ $=$ Thalamic area, $\mathrm{HC}=$ Hippocampus, $\mathrm{CL}=$ Cerebellum, $\mathrm{RB}=$ Rest of brain. Fig. 11 in parentheses are tie corrected $\chi^{2}$ values obtained by Kruskal-Wallis

\section{DISCUSSION}

Unsaturated isoprenoid side chains with double bonds in the $3^{\prime}, 7^{\prime}$ and 11' positions in TTs made them a unique component of vitamin E. Naturally, TTs occur in esterified forms and available in many seed oils, however, in low quantities [19]. Following oral exposure, after being cleaved by esterase enzymes, TTs are absorbed in small intestine along with absorption of other fats. Then TTs are packed in chilomicrons and transported into the lymphatic system [3]. The bioavailability of TT after oral exposure depends on the type of constituent isomers-being it highest in $\alpha$-TT [2]. Most of their antioxidant activities are performed at the plasma as they encounter the oxidative free radicals during their stay in blood stream [3]. However, it takes about $2 \mathrm{~h}$ to reach the peak value of plasma TT after oral exposure and the half-life of TT in plasma is only 3.5h [2]. Plasma TTs are taken up by the tissues, either with the help of lipoprotein lipases or through receptor-mediated endocytosis of lipoprotein [3] and eventually, TTs are removed from the plasma within 24h [2]. While adipose tissues, adrenal glands, heart, pancreas, skeletal muscles are the major taker of TTs, the brain receives only nominal amount $[20,21]$.

The abundance of easily oxidized lipid along with a greater rate of oxygen consumption renders brain an assailable target of oxidative insult [22]. Approximately $2 \%$ of the oxygen consumed by the brain (nearly 20\% of the overall consumption) is employed for the generation of free radicals [23]. These hyper-reactive oxygen and nitrogen species may facilitate the process of cell death by hampering the critical cellular functions [22]. While the brain is not well defended by the antioxidants overall, some areas like hippocampus and cerebellum have a lower endogenous level of vitamin E [24]. It is expected that oral supplementation of TT should improve the oxidant handling capacities of brain regions, as already suggested [25].
Oral supplementation with TT for two weeks has improved the weekly growth of rats transiently; accordingly, significant differences in body weights are observed. Although, Radhakrishnan et al. [26] reported no significant deviation in body weight between the TT-treated and control groups, the difference in observations may be due to divergence in the dose of TT used. Radhakrishnan et al. [26] have used $5 \mathrm{mg}$ of $\gamma \mathrm{TT} / \mathrm{Kg}$ body weight for $24 \mathrm{~d}$, while $10 \mathrm{mg}$ of TT is orally fed to each rat each day for $14 \mathrm{~d}$ in the current experiment. Interestingly, the groups of rats did not differ in terms of food intake, while the $\mathrm{TT}_{+}$group has consumed the noticeably higher amount of water starting from post-TT treatment. Observed higher water intake and increased growth are in conformity with the earlier observations made by Budin et al. [27] where they have observed increased fluid uptake and prevention of body weight loss in diabetic rats under the influence of TT rich fraction supplementation.

Only insignificant differences between the $\mathrm{TT}_{+}$and $\mathrm{TT}_{0}$ groups have been observed in serum GSH and TBARS levels in different times of treatment protocol. Nevertheless, elevated GSH has been noted by Radhakrishnan et al. [26] after $24 \mathrm{~d}$ of $\gamma \mathrm{TT} \quad(5 \mathrm{mg} / \mathrm{Kg})$ supplementation. On the other hand, no improvement in serum vitamin $\mathrm{C}$ and TBARS levels is observed in human subjects, even after $24 \mathrm{w}$ of supplementation with TT-rich-fraction (TRF) of palm oil [27].

In the same human trial, Jubri et al. [28] have also reported unaltered activities of erythrocyte SOD, GPx and catalase till $12 \mathrm{w}$ of TRF supplementation, while the activities of SOD and GPx remain similar till $24 \mathrm{w}$ of TRF supplementation; decrease in catalase activity has been noticed. Radhakrishnan et al. [26] also have reported insignificant alterations in the serum SOD activity after 24 $\mathrm{d}$ of oral supplementation $\gamma \mathrm{TT}(5 \mathrm{mg} / \mathrm{Kg})$. No alteration in activities of serum catalase, SOD, GPx and GR has been noticed in any of the tested time points during the current study. In a study with palm 
vitamin E (200 mg/Kg bw p. o. four weeks) on rats, Matough et al. [29] have noticed no change in total antioxidant capacity of plasma (Ferric reducing antioxidant power assay) even though GSH and oxidized glutathione (GSSG) contents have been raised significantly in erythrocytes. Nevertheless, this study reports only transient significant decrease in GI-SPHC during immediately after the completion of TT supplementation, which may be related to the observed statistically insignificant but noticeable decrease in serum catalase activity during the same time.

Interestingly, none of the brain regions has demonstrated significant alterations of catalase activities at the completion of $4 \mathrm{w}$ of the postTT-supplementation period in the current study. It is also noteworthy that neither the GI-SPHC nor the GD-SPHC has been found to alter significantly, even though SOD and GPx activities in specific brain regions have been estimated to vary significantly from their respective counterparts.

Notwithstanding the shortcomings of the present study regarding the level of oxidative stress parameters of brain regions immediately after the TT supplementation, it can be concluded that the effect of TT supplementation with current dose and duration could impact the brain oxidative stress parameters in a region-specific manner even $4 \mathrm{w}$ after the completion of TT supplementation; however, this is not true for the regional SPHCs of the brain.

\section{ACKNOWLEDGEMENT}

This work is financially supported (No. ERIP/ER/1204652/M01/ 1496) by the Directorate of Extramural Research and Intellectual Property Rights (ER and IPR), Defence Research and Development Organization (DRDO), Government of India. Oryza Oil and Fat Chemical Co. Ltd., Japan, has provided the Tocotrienol@-90 sample to carry out this work. The authors wish to thankfully acknowledge the support received from the Management of NRI Academy of Sciences to carry out the work.

\section{CONFLICT OF INTERESTS}

\section{Declared none}

\section{REFERENCES}

1. Kaneai N, Sumitani K, Fukui K, Koike T, Takatsu H, Urano S. Tocotrienol improves learning and memory deficit of aged rats. J Clin Biochem Nutr 2016;58:114-21.

2. Aggarwal BB, Sundaram C, Prasad S, Kannappan R. Tocotrienols, the vitamin E of the 21st century: its potential against cancer and other chronic diseases. Biochem Pharmacol 2010;80:1613-31.

3. Ahsan H, Ahad A, Iqbal J, Siddiqui WA. Pharmacological potential of tocotrienols: a review. Nutr Metab (Lond) 2014;11:52.

4. Azlina MF, Nafeeza MI, Khalid BA. A comparison between tocopherol and tocotrienol effects on gastric parameters in rats exposed to stress. Asia Pac J Clin Nutr 2005;14:358-65.

5. Reznick AZ, Witt E, Matsumoto M, Packer L. Vitamin E inhibits protein oxidation in skeletal muscle of resting and exercised rats. Biochem Biophys Res Commun 1992;189:801-6.

6. Nayak P, Sharma SB, Chowdary NVS. Pro-oxidant status based alterations in cerebellar antioxidant response to aluminum insult. Neurochem J 2012;6:44-52.

7. Nayak P, Sharma SB, Chowdary NV. Aluminum and ethanol induce alterations in superoxide and peroxide handling capacity (SPHC) in the frontal and temporal cortex. Indian J Biochem Biophys 2013;50:402-10.

8. Nagapan G, Meng Goh Y, Shameha Abdul Razak I, Nesaretnam $\mathrm{K}$, Ebrahimi M. The effects of prenatal and early postnatal tocotrienol-rich fraction supplementation on cognitive function development in male offspring rats. BMC Neurosci 2013;14:77.

9. Stevens L. Buffers and the determination of protein concentration. In: Eisenthal R, Danson MJ. editors. Enzymatic Assay-A Practical Approach. Oxford: IRL Press; 1992. p. 316-35.

10. Griffith OW. Determination of glutathione and glutathione disulfide using glutathione reductase and 2-vinylpyridine. Anal Biochem 1980;106:207-12.
11. Buege JA, Aust SD. Lipid peroxidation. Methods Enzymol 1978;51:302-10.

12. Sinhuber RO, Yu TC, Yu TC. Characterization of the red pigment formed in the thiobarbituric acid determination of oxidative rancidity. Food Res 1958;23:626-30.

13. Marklund S, Marklund G. Involvement of superoxide anion radical in the autoxidation of pyrogallol and a convenient assay for superoxide dismutase. Eur J Biochem 1974;47:469-74.

14. Beers RF Jr, Sizer IW. A spectrophotometric method for measuring the breakdown of hydrogen peroxide by catalase. J Biol Chem 1952;195:133-40.

15. Paglia DE, Valentine WN. Studies on the quantitative and qualitative characterization of erythrocyte glutathione peroxidase. J Lab Clin Med 1967;70:158-69.

16. Pinto RE, Bartley W. The effect of age and sex on glutathione reductase and glutathione peroxidase activities and on aerobic glutathione oxidation in rat liver homogenates. Biochem J 1969;112:109-15.

17. Nayak P. Prooxidative and antioxidative intervention of aluminum-induced oxidative stress and neurodegeneration. $\mathrm{PhD}$ Thesis. Chettinad Academy of Research and Education; 2014.

18. Hammer O, Harper DAT, Ryan PD. PAST: Paleontological statistics software package for education and data analysis. Palaeontologia Electronica; 2001. Available from: http://palaeo-electronica.org/2001_1/past/issue1_01.htm. [Last accessed on 20 Sep 2016]

19. Aan GJ, Sarmugam AS, Karim NA. Effect of tocotrienol-rich fraction (TRF) on synpatogenic molecules in aging caenorhbdis elegans. Int J Biomed Advan Res 2015;6:36-42.

20. Hayes KC, Pronczuk A, Liang JS. Differences in the plasma transport and tissue concentrations of tocopherols and tocotrienols: observations in human and hamsters. Proc Soc Exp Biol Med 1993;202:353-9.

21. Kawakami Y, Tsuzuki T, Nakagawa K, Miyazawa T. Distribution of tocotrienols in rats fed a rice bran tocotrienol concentrate. Biosci Biotechnol Biochem 2007;71:464-71.

22. Selvaraju TR, Khaza'ai H, Vidyadaran S, Abd Mutalib MS, Vasudevan R. The neuroprotective effects of tocotrienol rich fraction and alpha-tocopherol against glutamate injury in astrocytes. Bosnian J Basic Med Sci 2014;14:195-204.

23. Sue-Mian T, Ngah WZW, Top GM, Mazlan M. Comparison of the effects of $\alpha$-tocopherol and $\gamma$-tocotrienol against oxidative stress in two different neuronal cultures. Sains Malaysiana 2010;39:145-56.

24. Tiwari V, Arora V, Chopra K. Attenuation of NF- $\kappa \beta$ mediated apoptotic signalling by tocotrienol ameliorates cognitive deficits in rats postnatally exposed to ethanol. Neurochem Int 2012;61:310-20.

25. Dasari P, Anandamurali R, Nayak P. Tocotrienol opposes the effect of light to moderate ethanol exposures in elevated plus maze performances of rats. Asian J Pharm Clin Res 2016;9:1-6.

26. Radhakrishnan A, Tudawe D, Chakravarthi S, Chiew GS, Haleagrahara N. Effect of $\gamma$-tocotrienol in counteracting oxidative stress in joint damage in collagen-induced arthritis in rats. Exp Ther Med 2014; 7:1408-14.

27. Budin SK, Taib IS, Jayusman PA, Chiang HH, Ramalingam A, Ghazali AR, et al. Ameliorative effect of the palm oil tocotrienolrich fraction on brain oxidative stress in fenitrothionadministered rats. Sains Malays 2014;43:1031-6.

28. Jubri Z, Latif AA, Top AGM, Ngah WZW. Oxidative status of cigarette smokers with tocotrienol rich fraction supplementation. Middle-East J Sci Res 2014;21:897-904.

29. Matough FA, Budin SB, Hamid ZA, Louis SR, Alwahaibi N, Mohamed J. Plam vitamin E reduces oxidative stress and physical and morphological alterations of erythrocyte membranes in streptozotocin-induced diabetic rats. Oxid Antoxid Med Sci 2012;1:59-68.

\section{How to cite this article}

- Pitchaiah Dasari, Anandamurali R, Prasunpriya Nayak. Effect of tocotrienol pretreatment on ex vivo superoxide and peroxide handling capacities (SPHC) of rat serum and brain. Int J Pharm Pharm Sci 2017;9(3):116-122. 